\title{
Laparoscopic adrenalectomy: a report on 50 operations
}

Silvia Filipponi, Mario Guerrieri ${ }^{1}$, Giorgio Arnaldi, Marilena Giovagnetti, Ana M Masini, Emanuele Lezoche ${ }^{1}$ and Franco Mantero

Clinica di Endocrinologia, Universita' di Ancona, Ancona, Italy and ${ }^{1}$ Patologia Chirurgica, Universita' di Ancona, Ancona, Italy

(Correspondence should be addressed to F Mantero, Clinica di Endocrinologia, Ospedale di Torrette, Via Conca, 60020 Ancona, Italy)

\begin{abstract}
Objective: To investigate the feasibility, safety and results of laparoscopic transperitoneal adrenalectomies performed with the patient supine, in patients affected by secreting and silent adrenal lesions. Methods: Exclusion criteria were suspected adrenal primary malignancies. Fifty patients (33 women and 17 men; mean age 49.6 years, range 19-75 years) underwent 51 laparoscopic adrenalectomies (one bilateral). After complete endocrinological evaluation, computed tomography or magnetic resonance imaging, or a combination thereof, 14 non-secreting adenomas, 13 aldosterone-producing adenomas, 13 cortisol-producing adenomas, eight phaeochromocytomas (one bilateral), one androgen-secreting adenoma, and two metastases were considered eligible for adrenalectomy. In five patients, associated procedures were performed during surgery.

Results: The lesions ranged in size from 1.5 to $10 \mathrm{~cm}$. There were no intraoperative complications and no blood transfusions were required. The postoperative course was uneventful and painless in all patients. Mean postoperative hospital stay was 2.5 days. In all hypertensive patients, significant improvement or cure of hypertension was observed at follow-up (mean 18 months). In patients with secreting adenomas, normalization of hormone concentrations was obtained after removal of the tumour. In six patients with incidentaloma, the exaggerated 17-hydroxyprogesterone response to ACTH disappeared after surgery.

Conclusions: Secreting and non-secreting adrenal lesions were treated safely by laparoscopy. Relatively small incidentalomas and subclinical hormonally active tumours can be removed by laparoscopy. Early diagnosis enhances prevention and treatment.
\end{abstract}

European Journal of Endocrinology 138 548-553

\section{Introduction}

Routinely available biochemical tests for adrenal hormones and the widespread use of non-invasive imaging methods (ultrasound, computed tomography (CT) and magnetic resonance imaging (MRI)) have greatly improved the rate of early detection of adrenal masses, with obvious advantages in the evaluation and treatment of preclinical and overt hormonesecreting adrenal tumours and larger, progressing, non-functional adrenal masses that may require surgical removal (1).

The traditional surgical techniques to remove the adrenal glands are the anterior, posterior, flank and thoracoabdominal approaches, according to the size and endocrine function of the mass. Video-assisted resection of the adrenal glands has considerably changed the conventional surgical approaches and has extended the indications for adrenalectomy to considerably larger, non-hypersecreting masses.

Laparoscopic adrenalectomy was introduced into clinical practice by Gagner et al. in 1992 (2). These and other authors demonstrated that the techniques and the advantages of minimally invasive surgery could be extended to the adrenal approach (3-5).

Laparoscopic adrenalectomy is less painful than open adrenalectomy, which requires a large incision or the resection of the 12 th rib for the flank access $(6,7)$. This minimally invasive method makes it possible to reduce perioperative morbidity and to achieve faster recovery, and improves postoperative quality of life and cosmetic results $(3,8,9)$. The aim of this study was to evaluate the clinical results of 51 laparoscopic adrenalectomies performed in a heterogeneous group of patients suffering from secreting and silent adrenal lesions.

\section{Patients and methods}

Exclusion criteria were suspected adrenal primary malignancies. The degree of difficulty of the operation was evaluated by analysing the following parameters: gland diameter, side of the adenoma, operating time, number of trocars used, duration of hospital stay and pain-relieving medications administered. Operating time was calculated from the introduction to the 
removal of the laparoscope, and did not include the time required for positioning the patient on the operating table or that taken up by the lysis of existing adhesions.

From January 1994 until March 1997, 51 laparoscopic adrenalectomies (one bilateral) were performed in 50 patients by a single surgical team with wide experience in abdominal laparoscopy. The series included 33 women (mean age 48, range 21-75 years) and 17 men (mean age 51.2, range $19-73$ years).

Twenty-eight $(55 \%)$ of the lesions were located on the right side and $23(45 \%)$ on the left. A bilateral adrenalectomy was performed for a left recurrent phaeochromocytoma and a right phaeochromocytoma in a patient with multiple endocrine neoplasia (MEN 2A) who had been operated on by laparotomy for a left phaeochromocytoma 3 years earlier.

Seven $(14 \%)$ patients with right adrenal lesions had previously undergone abdominal operations (four appendectomies, one appendectomy and cholecystectomy, one appendectomy, cholecystectomy and hysterosalpingectomy, one subtotal colectomy), as had six $(12 \%)$ patients with left adrenal tumours (two appendectomies, one appendectomy and right ovariectomy, one left ovariectomy, one gastroduodenal resection, one total gastrectomy).

In five $(10 \%)$ patients with associated diseases (three gallbladder lithiasis, one gallbladder adenoma, one ovarian cyst), one right adrenalectomy and cholecystectomy, one right adrenalectomy and left ovariectomy, three left adrenalectomies and cholecystectomies were performed in the course of the laparoscopic procedure.

The associated operations did not require additional port sites, only a change in the tilt of the operating table.

The physical status classification of the American Society of Anesthesiology (ASA) was ASA I in 39 patients, II in 10 patients and III in one patient.

\section{Endocrinological evaluation}

Baseline hormonal data included the diurnal rhythm of plasma cortisol, urinary free cortisol, adrenocorticotrophic hormone (ACTH), dehydroepiandrosterone, 17-hydroxyprogesterone (17-OHP), testosterone, supine and upright plasma renin activity (PRA) and aldosterone, urinary aldosterone 18-glucuronide, catecholamines excretion and vanillin mandelic acid (VMA). Hormonal dynamic tests, dexamethasone suppression test (1 or $8 \mathrm{mg}$ overnight), corticotrophin-releasing hormone test, short ACTH stimulation test for cortisol and 17-OHP, and captopril test for PRA and aldosterone were also performed where appropriate. CT, MRI, or both, were performed in all patients. Twelve $(24 \%)$ patients underwent ${ }^{75} \mathrm{Se}$-cholesterol or ${ }^{131}$ I-metaiodobenzylguanidine ( $\left.\mathrm{I}^{131} \mathrm{MIBG}\right)$ radioisotopic scintiscans.

Complete hormonal tests were also performed in patients with incidentally detected adrenal masses.

The patients considered eligible for laparoscopic adrenalectomy are listed in Table 1. Of 51 tumours,
Table 1 Characteristics of 50 patients eligible for laparoscopic adrenal surgery. Values are mean or mean (range).

\begin{tabular}{lccc}
\hline Diagnosis & $\boldsymbol{n}$ & $\begin{array}{c}\text { Size } \\
(\mathrm{cm})\end{array}$ & $\begin{array}{c}\text { Age } \\
\text { (years) }\end{array}$ \\
\hline Incidentaloma & 14 & $\begin{array}{c}4.6 \\
(3.5-7)\end{array}$ & 60.2 \\
Aldosterone-producing adenoma & 13 & $\begin{array}{c}4.2 \\
(2.5-5.5)\end{array}$ & 46.8 \\
Cortisol-producing adenoma & 13 & $\begin{array}{l}4.9 \\
(2.5-10)\end{array}$ & 40.8 \\
Phaeochromocytoma (one bilateral) & 8 & 5 & 37.8 \\
Androgen-secreting adenoma & 1 & $\begin{array}{l}(1.5-8.5) \\
1.7\end{array}$ & 74 \\
Metastases & 2 & 6.0 & 58 \\
& & $(5.5-6.5)$ & \\
\hline
\end{tabular}

$n$, number of cases.

16 were detected incidentally: 10 non-hypersecreting adenomas, one phaeochromocytoma, two metastases and three cortisol-secreting adenomas with preclinical Cushing's syndrome (10-12).

The indications for laparoscopic excision of nonhypersecreting adenoma included any mass of $4.0 \mathrm{~cm}$ or larger, especially in young patients, and lesions smaller than $4 \mathrm{~cm}$ that increased in diameter at imaging during follow-up.

Exclusion criteria were suspected malignancies suggested by irregular or lobulated margins, dishomogeneous density on CT scan, increased intensity on T2-weighted MRI compared with liver and isointensity with liver on T1-weighted MRI.

One patient with an aldosterone-producing adenoma was affected by von Willebrand's syndrome and required pre- and postoperative infusion with desmopressin (16 $\mu \mathrm{g} /$ day) $2 \mathrm{~h}$ before surgery and for 7 days after operation.

Control of hypertension was achieved before operation: patients with phaeochromocytoma were treated with $\alpha$-adrenergic blockade (doxazosin), occasionally associated with $\beta$-adrenergic blockade (propanolol); patients with hyperaldosteronism were treated with potassium kanrenoate.

All patients received short-term antibiotic prophylaxis (first-generation cephalosporin) during the operation. Patients operated on for cortisol-producing adenoma or incidentaloma underwent substitutive therapy with hydrocortisone before and during surgery, and with cortisone acetate thereafter for different periods according to their individual needs. All patients received clinical and hormonal evaluation 7-30 days after discharge from hospital, and were then recalled periodically for follow-up.

\section{Surgical technique}

The patients were operated on in the supine position. Four 12-mm trocars (Ethicon Endo-Surgery, Cincinnati, 


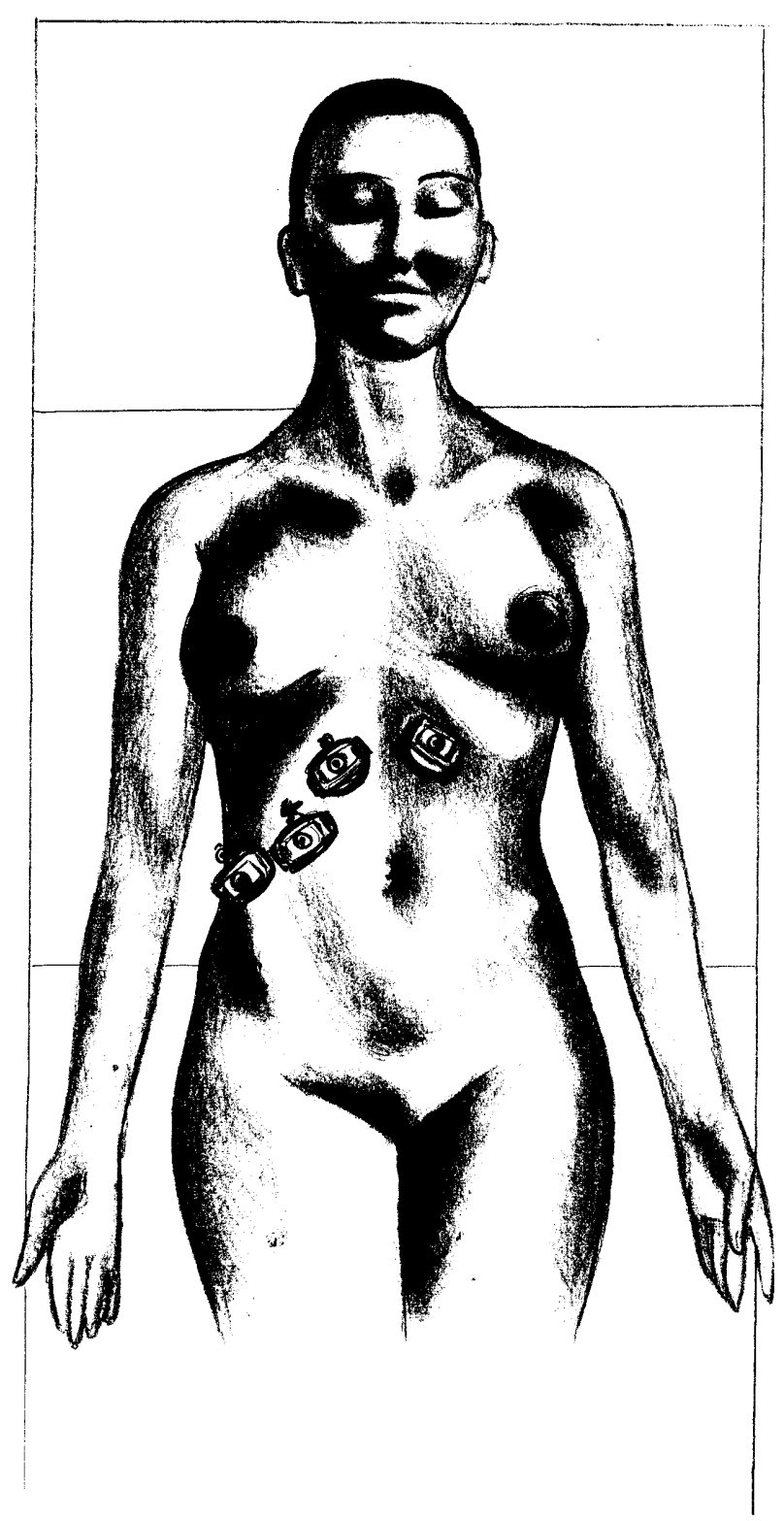

Figure 1 Trocar site position in right adrenalectomy, with the patient in the supine position.

$\mathrm{OH}$, USA) were used, as shown in Fig. 1. On the right side, the operation was begun by dividing the peritoneal reflection along the lateral side of the inferior vena cava. Blunt tissue dissection enabled identification of the transversely oriented short adrenal vein, which was closed with clips and cut. The gland was mobilized by blunt dissection and electrocautery of its small arteries and introduced into a specimen retrieval bag for removal.

On the left side, the splenic flexure of the colon was mobilized medially by dividing the peritoneal reflection along the left paracolic gutter and the splenocolic ligament, using sharp dissection and electrocautery.
Exposure of the adrenal gland was obtained by raising the stomach and the tail of the pancreas. The left adrenal vein was dissected, closed with clips and cut. Next, the small adrenal arteries were dissected and electrocoagulated. After its superior pole had been dissected, the gland was excised and removed.

\section{Results}

The lesions ranged from 1.5 to $10 \mathrm{~cm}$ in diameter (mean $4.8 \mathrm{~cm})$.

There were no intraoperative complications or mortality. Conversion to open surgery was never necessary, even though three patients required a prolonged adhesiolysis. None of the 50 patients required intra- or postoperative blood transfusions. One patient with phaeochromocytoma received intraoperativemedical therapy for hypertension and arrhythmia.

Mean operating time was $92 \mathrm{~min}$ for right adrenalectomies (range 58-180 min), $136 \mathrm{~min}$ for left adrenalectomies (range 90-220 min) and $280 \mathrm{~min}$ for the bilateral adrenalectomy (Table 2). Operating time improved over the course of the study, reflecting a learning curve: from $114 \mathrm{~min}$ for the first 10 right adrenalectomies to $82 \mathrm{~min}$ for the last 10 , and from $152 \mathrm{~min}$ for the first 10 left adrenalectomies to $118 \mathrm{~min}$ for the last 10 .

Postoperative pain was minimal and drugs for pain relief were administered to eight patients (16\%). Patients were able to ambulate $12-24 \mathrm{~h}$ after the operation and were allowed a liquid diet on the first day after surgery. All were discharged 2-3 days after operation (mean hospital stay 2.5 days) and resumed their normal activities within 7 days of the operation.

Mean follow-up time was 18 months (range 1-38 months). The two patients operated on for a single metastasis (from melanoma and gastric cancer) underwent chemotherapeutic treatment at the oncology centre: they were alive at 4 and 6 months of followup, respectively. No surgical complications arose during follow-up.

Before surgery, hypertension was present in 29 (58\%) of the patients (four with incidentaloma, eight with Cushing's syndrome, one with pre-Cushing's syndrome, 13 with aldosterone-producing adenoma, three with phaeochromocytoma). Significant improvement (15

Table 2 Correlation between localization of the lesions, operating time and hospital stay after laparoscopic adrenalectomy. Values are mean or mean (range).

\begin{tabular}{lccc}
\hline Side & $\boldsymbol{n}$ & $\begin{array}{c}\text { Operating time } \\
(\mathrm{min})\end{array}$ & $\begin{array}{c}\text { Hospital stay } \\
\text { (days) }\end{array}$ \\
\hline Right & 27 & $92(58-180)$ & 2.5 \\
Left & 22 & $136(90-220)$ & 2.5 \\
Bilateral & 1 & 280 & 3.0 \\
\hline
\end{tabular}

$n$, number of patients. 
Table 3 Hormone concentrations before and after laparoscopic adrenal surgery. Values are mean (range).

\begin{tabular}{|c|c|c|c|}
\hline & Normal range & Before operation & After operation \\
\hline \multicolumn{4}{|l|}{ Cushing's syndrome $(n=10)$} \\
\hline Plasma cortisol (nmol/l) & $140-700$ & $\begin{array}{l}728 \\
(448-1008)\end{array}$ & $\begin{array}{l}212^{*} \\
(28-476)\end{array}$ \\
\hline ACTH (ng/l) & $<60$ & $\begin{array}{l}12.1 \\
(5.6-15)\end{array}$ & $\begin{array}{l}46.8^{*} \\
(17-90)\end{array}$ \\
\hline Urinary free cortisol (nmol/day) & $98-378$ & $\begin{array}{l}1478.4 \\
(355.6-2800) \\
36.1 \dagger\end{array}$ & $\begin{array}{l}7.0 \dagger \\
(0-42)\end{array}$ \\
\hline \multicolumn{4}{|l|}{ Pre-Cushing's syndrome $(n=3)$} \\
\hline Urinary free cortisol (nmol/day) & $98-378$ & $\begin{array}{l}630 \\
(560-756)\end{array}$ & $\begin{array}{l}7.0 \dagger \\
(0-42) \\
330.4 \ddagger\end{array}$ \\
\hline \multicolumn{4}{|l|}{ Aldosterone-producing adenoma $(n=13)$} \\
\hline Urinary aldosterone (nmol/day) & $17-42$ & $\begin{array}{l}115 \\
(40-229)\end{array}$ & $\begin{array}{l}34.6 \S \\
(6-45)\end{array}$ \\
\hline Supine plasma aldosterone $(\mathrm{nmol} / \mathrm{l})$ & $0.02-0.4$ & $\begin{array}{l}1.06 \\
(0.55-1.37)\end{array}$ & $\begin{array}{l}0.09 \ddagger \\
(0.06-0.16)\end{array}$ \\
\hline Upright plasma aldosterone (nmol/l) & $0.11-0.8$ & $\begin{array}{l}1.13 \\
(0.73-1.32)\end{array}$ & $\begin{array}{l}0.13 \ddagger \\
(0.06-0.22)\end{array}$ \\
\hline
\end{tabular}

* 6 months after surgery; $\uparrow 1$ month after surgery; $¥ 8.5$ months after surgery; $§ 3$ months after surgery.

patients) or cure (14 patients) of hypertension was observed 3 months after the operation. After adrenalectomy, 15 patients required a single-drug regimen to control their hypertension, whereas before surgery they had required two or three medications.

Before the operation, urinary catecholamine concentrations were increased in three patients with phaeochromocytomas, but they were normal in all patients 3 months after operation.

Of the 10 patients with overt Cushing's disease, none was affected by severe obesity or diabetes. The body mass index was normal in six of them and slightly increased in four, while eight of these patients exhibited the characteristic (abdominal, supraclavicular) fat deposition. Nine patients with Cushing's syndrome were hypertensive; of two oligoamenorrhoeic patients, one was affected by glaucoma and one was operated on for cutaneous melanoma; cure of oligoamenorrhoea and glaucoma was observed after surgery. In all patients with cortisol- and aldosterone-producing adenoma, normalization of hormonal data was achieved after operation (Table 3).

After ACTH, 17-OHP was evaluated in seven patients with incidentally discovered adrenocortical adenomas (four non-hypersecreting and three pre-Cushing's syndrome), and was found to be increased in six of them: mean after $60 \mathrm{~min}$ was $36.6 \mathrm{nmol} / \mathrm{l}$ (mean basal value $3.9 \mathrm{nmol} / \mathrm{l}$ ). In these six patients, the exaggerated 17-OHP response disappeared after surgery (mean after $60 \mathrm{~min}$ : $6.06 \mathrm{nmol} / \mathrm{l}$; mean basal value: $3.33 \mathrm{nmol} / \mathrm{l}$ ).

\section{Discussion}

The available options for laparoscopic removal of the adrenal gland are: anterior access with the patient in supine position, flank approach with the patient in lateral decubitus, and posterior approach (retroperitoneal). We chose the transperitoneal approach with the patient supine for its satisfactory exposure of the anatomical structures (adrenal veins, vena cava, etc.) and good control of the bleeding, and because it allows associated surgical procedures to be performed. Although the flank and posterior approaches are less invasive and require less dissection on the left side, the patient's position prevents the performance of associated surgical procedures, exploration of the contralateral gland or immediate conversion to open surgery in case of bleeding. In the present cases, there were no operative complications, and conversion to open surgery was never required.

Lesion size did not influence the procedure and an adrenal tumour $10 \mathrm{~cm}$ in diameter was removed successfully. Previous abdominal surgery may impose a long and difficult dissection of the adhesions. We performed five laparoscopic adrenalectomies in patients who had undergone previous upper abdominal surgery; this is not a contraindication if the surgeon has extensive experience in laparoscopic abdominal procedures. Even a coagulopathy was not considered an absolute contraindication to laparoscopy: we treated one woman affected by von Willebrand's syndrome with administration of desmopressin, without complications. In contrast, the presence of an invasive adrenal carcinoma with suspected vascular infiltration and nodal metastases did contraindicate the laparoscopic approach.

The transperitoneal approach and the simple tilting of the operating table allowed us to treat associated pathologies such as cholelithiasis and ovarian cysts during laparoscopy. We performed associated procedures 
through the same port sites as were used for adrenalectomy. This technique makes it possible to explore the contralateral gland and, if necessary, excise bilateral adrenal lesions without repositioning the patient. Indeed, among the major advantages of transperitoneal laparoscopic adrenalectomy are the ability to obtain magnified views of the structures of the abdominal cavity and to use intraoperative ultrasound $(2,5)$. In our series of patients, we were able to discover small lesions of the abdominal organs and to perform biopsies.

Mean operating time was satisfactory, and shorter in the last 20 of the adrenalectomies, in accordance with the learning curve of the surgical team. Operating time for the bilateral adrenalectomy was acceptable ( $280 \mathrm{~min})$. The time required to dissect the adhesions in patients with previous abdominal operations was also acceptable and ranged between 30 and $60 \mathrm{~min}$. Intra- and postoperative bleeding were minimal and no patient needed blood transfusions.

The laparoscopic approach provides better exposure of the anatomical structures than open surgery, especially for the adrenal vein, which can be closed early, before extensive mobilization and manipulation of the adrenal gland and tumour $(6,7)$. This technical aspect was essential in the cases of phaeochromocytoma (14). Despite preoperative pharmacological treatment, one patient with phaeochromocytoma required intraoperative therapy for hypertension and cardiac arrhythmia.

Postoperative pain was minimal and required drug administration only in $16 \%$ of patients. The laparoscopic approach allowed early oral feeding and quick mobilization, without postoperative complications such as bronchopneumonia, wound infections and deep vein thrombosis. Recently, other authors have demonstrated better postoperative quality of life and fewer postoperative complications after adrenal laparoscopic surgery than after open adrenalectomy $(6,7,15)$.

Mean hospital stay was brief, even though, as a precaution, we postponed the discharge of the first few patients to the 4 th or 5 th days. All patients were able to resume their normal activities within 7 days of the operation.

Mean follow-up time was 18 months (range 1-38). No surgical complications arose in this period.

Improvement or cure of most of the clinical symptoms caused by hormonal hypersecretion were observed at follow-up, as was gradual recovery of normal function of the remaining gland in the cases of unilateral adrenal hyperfunctioning adenomas.

The laparoscopic approach also appears to be suitable for surgical treatment of adrenal masses that are detected incidentally by ultrasound, CT or MRI performed for reasons other than suspected adrenal disease. These incidentalomas require diagnostic and therapeutic attention; they are usually benign, but up to $10-20 \%$ of them may be primary or secondary malignancies (16). Moreover, most tumours that appear clinically silent are in fact often found to secrete discrete amounts of cortisol, and to be phaeochromocytomas or aldosteronomas (17).

In overt Cushing's syndrome, adrenalectomy may be required to avoid morbidity or to improve metabolic and cardiovascular disorders that may be related to the hormonal activity (obesity, insulin resistance, diabetes, hypertension) $(18,19)$, but the indication for surgery in patients with subtle abnormalities of cortisol secretion (so-called pre-Cushing's syndrome) is still debated. In elderly and asymptomatic patients, with non-functioning and non-growing adrenal adenomas, adrenalectomy is probably not indicated. However, the reduction in blood pressure values observed after the removal of tumours classified as silent in our series is intriguing and leaves open the question of subtle, non-detectable hormonal abnormalities.

The normalization of the 17-OHP response to ACTH in non-secreting tumours after the operation shows that this peculiar steroid pattern is less likely to be a marker of a congenital enzymatic disorder evolving in adrenal nodules (20), but rather a sign of disrupted intratumoral steroidogenesis $(13,21)$. Research (e.g. molecular analysis of the 21-hydroxylase gene in tumoral and genomic DNA) is in progress to challenge the hypothesis of somatic intratumoral acquired defects.

In conclusion, our experience demonstrates that laparoscopic adrenal surgery is an effective and safe method to treat secreting and non-secreting masses. Relatively small adrenal incidentalomas suspected of malignancy, exhibiting growth at follow-up imaging, or presenting with a slightly abnormal hormonal pattern can be removed by minimally invasive procedures, with early diagnosis enhancing the success of prevention and treatment of clinically related symptoms.

\section{Acknowledgements}

This work was supported in part by grant AIRC.

\section{References}

1 Ambrosi B, Passini E, Re T \& Barbetta L. The clinical evaluation of silent adrenal masses. Journal of Endocrinological Investigation 199720 90-107.

2 Gagner M, Lacroix A \& Prinz RA. Early experience with laparoscopic approach for adrenalectomy. Surgery 1993114 $1120-1125$

3 Gagner M, Lacroix A, Bolte E \& Pomps A. Laparoscopic adrenalectomy. Surgical Endoscopy 19937 122-125.

4 Deans GT, Kappadia R, Wedgewood K, Royston CMS \& Brough WA. Laparoscopic adrenalectomy. British Journal of Surgery 199582 994-995.

5 Stoker ME, Patwardhan N \& Maini BS. Laparoscopic adrenal surgery. Surgical Endoscopy 19959 387-391.

6 Brunt LM, Doherty GM, Norton JA. Soper NJ, Ouasebarth MA \& Moley JF. Laparoscopic adrenalectomy compared with open adrenalectomy for benign adrenal neoplasms. Journal of American College of Surgery 1996183 1-10.

7 Prinz RA. A comparison of laparoscopic and open adrenalectomies. Archives of Surgery 1995130 489-494. 
8 De Canniere L, Lorge F, Rosiere A, Joucken K \& Michel LA. From laparoscopic training on an animal model to retroperitoneoscopic or coelioscopic adrenal and renal surgery in human. Surgical Endoscopy 19959 699-701.

9 Mercan S, Seven R, Ozarmagan S \& Tezelman S. Endoscopic retroperitoneal adrenalectomy. Surgery 1995118 1071-1076.

10 Bernini GP, Vivaldi MS, Argenio GF, Moretti A, Sgrò M \& Salvetti A. Frequency of pheochromocytoma in adrenal incidentalomas and utility of the glucagon test for the diagnosis. Journal of Endocrinological Investigation 199720 65-71.

11 Glaz E, Racz K, Varga I, Kiss R, Toth M \& Futo L. Mineralocorticoid production of adrenal cortical adenomas. Journal of Steroid Biochemistry and Molecular Biology 199345 57-64.

12 Reincke M, Nieke J, Krestin GP, Saeger W, Allolio B \& Winkelmann W. Preclinical Cushing's syndrome in adrenal 'incidentalomas': comparison with adrenal Cushing's syndrome. Journal of Clinical Endocrinology and Metabolism 199275826 832.

13 Del Monte P, Bernasconi D, Bertolazzi L, Meozzi M, Badaracco B \& Torre R. Increased $17 \alpha$-hydroxyprogesterone response to ACTH in silent adrenal adenoma: cause or effect? Clinical Endocrinology $199542273-277$.

14 Meurisse M, Joris J, Hamoir E, Hubert B \& Charlier C. Laparoscopic removal of pheochromocytoma. Why? When? and Who? (reflections on one case report). Surgical Endoscopy 1995 9 431-436.

15 Van Wyk JJ, Gunther F, Ritzen EM, Wedell A, Cutler GB, Migeon C $\&$ New MI. Therapeutic controversies. The use of adrenalectomy as a treatment for congenital adrenal hyperplasia. Journal of Clinical Endocrinology and Metabolism 199681 3180-3190.

16 Angeli A, Osella G, Alì A \& Terzolo M. Adrenal incidentaloma: an overview of clinical and epidemiological data from the National Italian Study Group. Hormone Research 199747 279-283.

17 Mantero F, Masini AM, Opocher G, Giovagnetti M \& Arnaldi G. Adrenal incidentaloma: an overview of hormonal data from the National Italian Study Group. Hormone Research 199747 284-289.

18 Go H, Takeda M \& Imai T. Laparoscopic adrenalectomy for Cushing' s syndrome: comparison with primary aldosteronism. Surgery $199511711-17$.

19 Van Heerden JA, Young WF Jr, Grant CS \& Corpenter PC. Adrenal surgery for hypercortisolism: surgical aspects. Surgery 1995117 466-472.

20 Jaresch S, Kornely E, Kley HK \& Schlaghecke R. Adrenal incidentaloma and patients with homozygous or heterozygous congenital adrenal hyperplasia. Journal of Clinical Endocrinology and Metabolism 199274 685-689.

21 Racz K, Pinet F, Marton T, Szende B, Glaz E \& Corvol P. Expression of steroidogenic enzyme messenger ribonucleic acids and corticosteroid production in aldosterone-producing and 'nonfunctioning' adrenal adenomas. Journal of Clinical Endocrinology and Metabolism 199377 677-682.

Received 4 August 1997

Accepted 26 January 1998 